Mathematical Sciences and Applications

E-NOTES

MATHEMATICAL

SCIENCES

AND APPLICATIONS

E-NOTES

https://doi.org/10.36753 /msaen.981131

10 (3) 146-158 (2022) - Research Article

ISSN: 2147-6268

(c)MSAEN

\title{
A Finite Difference Approximation for Numerical Simulation of 2D Viscous Coupled Burgers Equations
}

\author{
Nuri Murat Yağmurlu* and Abdulnasır Gagir
}

\begin{abstract}
Many of the physical phenomena in nature are usually expressed in terms of algebraic, differential or integral equations.Several nonlinear phenomena playing a very important role in engineering sciences, physics and computational mathematics are usually modeled by those non-linear partial differential equations (PDEs). It is usually difficult and problematic to examine and find out nalytical solutions of initial-boundary value problems consisting of PDEs. In fact, there is no a certain method or technique working well for all these type equations. For this reason, their approximate solutions are usually preferred rather than analytical ones of such type equations. Thus, many researchers are concentrated on approximate methods and techniques to obtain numerical solutions of non-linear PDEs. In the present article, the numerical simulation of the two-dimensional coupled Burgers equation (2D-cBE) has been sought by finite difference method based on Crank-Nicolson type approximation. Widely used three test examples given with appropriate initial and boundary conditions are used for the simulation process. During the simulation process, the error norms $L_{2}, L_{\infty}$ are calculated if the exact solutions are already known, otherwise the pointwise values and graphics are provided for comparison. The newly obtained error norms $L_{2}, L_{\infty}$ by the presented schemes are compared with those of some of the numerical solutions in the literature. A good consistency and accuracy are observed both by numerical values and visual illustrations.
\end{abstract}

Keywords: 2D viscous Burgers equation; Crank-Nicolson;Finite difference method.

AMS Subject Classification (2020): Primary: 35Q51 ; Secondary: 74J35, 33F10.

${ }^{*}$ Corresponding Author

\section{Introduction}

Most of the natural principles and laws in the real world are modelled by differential equations and only a few of them could be solved analytically. Thus obtaining numerical solutions for those equations has become more important. Throughout history of mankind, scientists and engineers have utilized mathematics for describing the physical characteristics of the universe by searching appropriate modelling methods and techniques. In this context, it is seen that numerous fundamental phenomena in ecology, physics, finance, data science, mechanical engineering 
etc. could be modelled by means of various differential equations. Thus, finding analytical and numerical solutions of those differential equations has become a significant part of scientific studies. Becuase of this fact, over the years, a wide range of efficient and effective methods have been proposed and developed for solving those equations. Among others, one of those differential equations is the two-dimensional coupled Burgers equation (2D-cBE). There are many theoretical and numerical studies about the 2D-cBE equation in the literature. Fletcher [1] has presented its exact solution via applying the two-dimensional Hopf-Cole transform to the 2D coupled Burgers equation. 2D-cBE has been solved approximately by many researchers using several methods. For instance, Yagmurlu and Gagir [2] have sought the numerical solution of the two-dimensional coupled Burgers equation by finite difference method based on Rubin-Graves type linearization. Fletcher [3] has made a study to compare finite difference and finite element methods. Goyon [4] applied multi level alternating direction implicit methods. Ali et al. [5] have utilized collation method based on the radial base functions. Jain and Holla [6] have proposed two different schemes based on the cubic spline basis function. Bahadır [7] has solved the problem by means of fully implicit finite difference method. Khater et al. [8] have put forward the approximate solution of some Burgers type nonlinear partial differential equations using Chebyshev spectral collocation method. Mittal and Jiwari [9] have used the differential quadrature method using the Chebyshev-Gauss-Lobatto nodal points. Liao [10] obtained the numerical solution of the two-dimensional coupled Burgers equation by solving the two-dimensional linear heat equation obtained by applying the two-dimensional Hopf-Cole transformation to the two-dimensional coupled Burgers equation using the fourth-dimensional finite difference method. Zhu et al. [11] applied the discrete Adomian decomposition method. Srivastava et al. have applied [12] Crank-Nicolson finite difference method, Tamsir and Srivastava [13] have used semi-implicit finite difference method, Srivastava and Tamsir [14] have utilized Crank-Nicolson semi-implicit finite difference method, Thakar and Wani [15] have used linear finite difference method, Srivastava et al. [16] have applied implicit logarithmic finite difference method, Srivastava et al. [17] have used implicit exponential finite difference method, Srivastava and Singh [18] have used explicit-implicit finite difference method, Zhang et al. [19] have used full finite difference and non-standard finite difference methods, Mittal and Tripathi [20] have applied modified bi-cubic B-spline collocation method, Tamsir et al. [21] have used exponential modified cubic-B-spline differential quadrature method, Zhanlav et al. [22] have applied high order explicit finite difference method, Chai and Ouyang [34] have used proper stabilized Galerkin methods, and Ngondiep [23] has utilized three-level explicit time-split MacCormack algorithm. Saqib et al. [24] have dealt with numerical solutions of 2-dimensional time dependent coupled non-linear systems. Wubs and Goede [25], in their article, considered the fully explicit method resulting from the truncation in the solution process and chosen one of the test examples as the 2-dimensional coupled Burgers' equation. Kutluay and Yagmurlu [26] have proposed and succesfully applied the modified bi-quintic B-spline base functions for the two dimensional unsteady Burgers' equation using the Galerkin method. Başhan [27] has solved coupled viscous Burgers' equations numerically in the presence of very large Reynolds numbers. Başhan et al. [28] have applied quintic B-spline differential quadrature method to find the numerical solution of the modified Burgers' equation. Uçar et al. [29] have aimed to obtain the numerical approximate solutions of the nonlinear modified Burgers' equation via the modified cubic B-spline differential quadrature methods. Karakoç et al. [30] have obtained a numerical solution of the modified Burgers' equation by using quartic B-spline subdomain finite element method over which the nonlinear term is locally linearized and using quartic B-spline differential quadrature method. Başhan. [31] has modified quintic B-spline base function to use for numerical solution of the Burgers' equation. Karakoç and Bhowmik [32] have studied solitary-wave solutions of the nonlinear Benjamin-Bona-Mahony-Burgers equation based on a lumped Galerkin technique using cubic B-spline finite elements for the spatial approximation. Bhowmik and Karakoç [33] have developed and analyzed a powerful numerical scheme for the nonlinear GRLW equation by Petrov-Galerkin method in which the element shape functions are cubic and weight functions are quadratic B-splines.

The present paper is outlined as follows: The first section presented the method based on Crank-Nicolson type approximation together with finite difference method and used for the numerical solution of two dimensional coupled Burgers equation. To test the efficiency and effectiveness of the method, the approximate solution of three model problems has been found out and given in tabular form by computing the nodal values and also the error norms $L_{2}$ and $L_{\infty}$ of the model examples of which the analytical solutions are known In addition, a comparison is made with the error norms of the numerical solutions obtained by several methods available in the literature. In the end of the article, a brief summary and plans about possible future studies are presented.

\section{A concise description of the Method}

In the present article, 2D coupled Burgers equation of the following form [13] 


$$
\begin{aligned}
& u_{t}+\mathrm{u} u_{x}+v u_{y}=\frac{1}{\operatorname{Re}}\left(u_{x x}+u_{y y}\right), \quad(x, y) \epsilon \Omega, t>0, \\
& v_{t}+\mathrm{u} v_{x}+v v_{y}=\frac{1}{\operatorname{Re}}\left(v_{x x}+v_{y y}\right), \quad(x, y) \epsilon \Omega, t>0,
\end{aligned}
$$

with the appropriate initial

$$
\begin{array}{ll}
u(x, y, 0)=\psi_{1}(x, y) ; & (x, y) \epsilon \Omega, \\
v(x, y, 0)=\psi_{2}(x, y) ; & (x, y) \in \Omega,
\end{array}
$$

and the boundary conditions

$$
\begin{array}{ll}
u(x, y, t)=\xi(x, y, t) ; & (x, y) \epsilon \partial \Omega \\
v(x, y, t)=\zeta(x, y, t) ; \quad(x, y) \epsilon \partial \Omega
\end{array}
$$

will be considered, where $u(x, y, t)$ and $v(x, y, t)$ are velocity components given over the solution domain of the problem $\Omega=\{(x, y): a \leqslant x \leqslant b, c \leqslant y \leqslant d\}$ together with its boundary $\partial \Omega . \psi_{1}, \psi_{2}, \xi$ and $\zeta$ are known smooth functions.Re is the Reynold number and $\varepsilon=\frac{1}{\mathrm{Re}}$. For the large values of the Reynold number, a shock wave having a cusp results in and numerical stability near this shock wave is nearly always difficult to obtain. This is obvious in many studies in the literature and also observed in the present study.

The solution domain of the problem in $x$-direction is divided into $N_{x}$ equal parts with length $h_{x}$, and in $y$-direction is divided into $N_{y}$ equal parts with length $h_{y}$ such that $x_{i}=a+i h_{x}, i=0(1) N_{x}$ and $y_{i}=c+j h_{y}$, $j=0(1) N_{y}$. As a result of these division processes, a rectangular grid is constructed over the solution domain of the problem by means of grid points $\left(x_{i}, y_{j}\right)$. For the time dimension, again a fixed step length $\Delta t$ is taken such that $t_{n}=n \Delta t, n=0(1) N$. Throughout the paper, the numerical computations are going to be carried out at each $t_{n}$ time step and the results are going to be obtained at the grid points of this rectangular grid. From now on, the numerical solutions of $u(x, y, t)$ and $v(x, y, t)$ at the grid point $\left(x_{i}, y_{j}, t_{n}\right)$ are going to be denoted by uppercase $U_{i, j}^{n}$ and $V_{i, j}^{n}$, respectively.

Now, 2D-cBE given as follows

$$
\begin{aligned}
& u_{t}+u u_{x}+v u_{y}=\frac{1}{\operatorname{Re}}\left(u_{x x}+u_{y y}\right), \\
& v_{t}+u v_{x}+v v_{y}=\frac{1}{\operatorname{Re}}\left(v_{x x}+v_{y y}\right),
\end{aligned}
$$

is first discretized by explicit finite difference method (EFDM)

$$
\begin{aligned}
U_{i, j}^{n+1} & =U_{i-1, j}^{n}\left(b_{1} U_{i, j}^{n}+a_{1}\right)+U_{i, j}^{n}\left(1-4 a_{1}\right)-U_{i+1, j}^{n}\left(b_{1} U_{i, j}^{n}-a_{1}\right)+U_{i, j-1}^{n}\left(b_{1} V_{i, j}^{n}+a_{1}\right) \\
& -U_{i, j+1}^{n}\left(b V_{i, j}^{n}-a_{1}\right), \\
V_{i, j}^{n+1} & =V_{i-1, j}^{n}\left(b_{1} U_{i, j}^{n}+a_{1}\right)+V_{i, j}^{n}\left(1-4 a_{1}\right)-V_{i+1, j}^{n}\left(b_{1} U_{i, j}^{n}-a_{1}\right)+V_{i, j-1}^{n}\left(b_{1} V_{i, j}^{n}+a_{1}\right) \\
& -V_{i, j+1}^{n}\left(b_{1} V_{i, j}^{n}-a_{1}\right),
\end{aligned}
$$

and then by implicit finite difference method (IFDM)

$$
\begin{aligned}
& -U_{i-1, j}^{n+1}\left(b_{1} U_{i, j}^{n}+a_{1}\right)+U_{i, j}^{n+1}\left(1+4 a_{1}\right)+U_{i+1, j}^{n+1}\left(b_{1} U_{i, j}^{n}-a_{1}\right)-U_{i, j-1}^{n+1}\left(b_{1} V_{i, j}^{n}+a_{1}\right) \\
& +U_{i, j+1}^{n+1}\left(b_{1} V_{i, j}^{n}-a_{1}\right)=U_{i, j}^{n}, \\
& -V_{i-1, j}^{n+1}\left(b_{1} U_{i, j}^{n}+a_{1}\right)+V_{i, j}^{n+1}\left(1+4 a_{1}\right)+V_{i+1, j}^{n+1}\left(b_{1} U_{i, j}^{n}-a_{1}\right)-V_{i, j-1}^{n+1}\left(b_{1} V_{i, j}^{n}+a_{1}\right) \\
& +V_{i, j+1}^{n+1}\left(b_{1} V_{i, j}^{n}-a_{1}\right)=V_{i, j}^{n},
\end{aligned}
$$


where $h_{x}=h_{y}, a_{1}=\varepsilon k / h_{x}^{2}=\varepsilon k / h_{y}^{2}, b_{1}=k / 2 h_{x}=k / 2 h_{y}$ and $\varepsilon=1 / \operatorname{Re}$ for $i, j=1(1) M-1$. When the average of EFDM and IFDM is taken, the following Crank-Nicolson finite difference schemes are obtained

$$
\begin{aligned}
& -U_{i-1, j}^{n+1}\left(d_{1} U_{i, j}^{n}+c_{1}\right)+U_{i, j}^{n+1}\left(1+4 c_{1}\right)+U_{i+1, j}^{n+1}\left(d_{1} U_{i, j}^{n}-c_{1}\right) \\
& -U_{i, j-1}^{n+1}\left(d_{1} V_{i, j}^{n}+c_{1}\right)+U_{i, j+1}^{n+1}\left(d_{1} V_{i, j}^{n}-c_{1}\right) \\
& =U_{i-1, j}^{n}\left(d_{1} U_{i, j}^{n}+c_{1}\right)+U_{i, j}^{n}\left(1-4 c_{1}\right)-U_{i+1, j}^{n}\left(d_{1} U_{i, j}^{n}-c_{1}\right) \\
& +U_{i, j-1}^{n}\left(d_{1} V_{i, j}^{n}+c_{1}\right)-U_{i, j+1}^{n}\left(d_{1} V_{i, j}^{n}-c_{1}\right),
\end{aligned}
$$

and

$$
\begin{aligned}
& -V_{i-1, j}^{n+1}\left(d_{1} U_{i, j}^{n}+c_{1}\right)+V_{i, j}^{n+1}\left(1+4 c_{1}\right)+V_{i+1, j}^{n+1}\left(d_{1} U_{i, j}^{n}-c_{1}\right) \\
& -V_{i, j-1}^{n+1}\left(d_{1} V_{i, j}^{n}+c_{1}\right)+V_{i, j+1}^{n+1}\left(d_{1} V_{i, j}^{n}-c_{1}\right) \\
& =V_{i-1, j}^{n}\left(d_{1} U_{i, j}^{n}+c_{1}\right)+V_{i, j}^{n}\left(1-4 c_{1}\right)-V_{i+1, j}^{n}\left(d_{1} U_{i, j}^{n}-c_{1}\right) \\
& +V_{i, j-1}^{n}\left(d_{1} V_{i, j}^{n}+c_{1}\right)-V_{i, j+1}^{n}\left(d_{1} V_{i, j}^{n}-c_{1}\right)
\end{aligned}
$$

where $h_{x}=h_{y}, c_{1}=\varepsilon k / 2 h_{x}^{2}=\varepsilon k / 2 h_{y}^{2} d_{1}=k / 4 h_{x}=k / 4 h_{y}$ and $\varepsilon=1 / \operatorname{Re}$ for $i, j=1(1) M-1$.Using the known $U^{n}$ and $V^{n}$ values in the finite difference schemes obtained as a result of this approximation, the unknown values of $U^{n+1}$ and $V^{n+1}$ at the desired time $t$ were obtained for all three model examples.

\section{Numerical Examples and Results}

In this section, the numerical solution of 2D coupled Burgers equation given by the equations (2.1)-(2.2), for three examples with appropriate initial and boundary conditions using the finite difference method based on Crank-Nicolson type approximation has been obtained. For all computations, the MATLAB software is used. In order to show the accuracy of the obtained numerical solutions, the following error norms $L_{2}$ and $L_{\infty}$ are calculated

To show how good the numerical results obtained by the present method, the error norms $L_{2}$ and $L_{\infty}$ given as

$$
L_{2}=\sqrt{\sum_{i=1}^{N_{x}-1} \sum_{j=1}^{N_{y}-1}\left|U_{i j}-\left(u_{\text {exact }}\right)_{i j}\right|^{2}},
$$

and

$$
L_{\infty}=\max _{i, j}\left|U_{i, j}-\left(u_{\text {exact }}\right)_{i, j}\right|
$$

where $u_{i j}^{n}$ are exact solutions and $U_{i j}^{n}$ are numerical solutions at the nodal points $\left(x_{i}, y_{j}, t_{n}\right)$ [35].The proposed numerical scheme is applied to the test problems and the computed approximate results for different values of the time step size $k$ and partition number $N$ at some values of $T$ on the solution domains are displayed in tables.

Example I: Firstly, finite difference method has been applied to 2D-cBE having the following exact solution over the region $\Omega=[0,1] \times[0,1][7]$

$$
\begin{aligned}
& u(x, y, t)=\frac{3}{4}-\frac{1}{4[1+\exp ((-4 x+4 y-t) \operatorname{Re} / 32]}, \\
& v(x, y, t)=\frac{3}{4}+\frac{1}{4[1+\exp ((-4 x+4 y-t) \operatorname{Re} / 32]} .
\end{aligned}
$$

Since the Example I has an exact solution, its initial and boundary conditions required for the application of the method are obtained from the analytical solution. Table (1) shows the numerical solutions of Example I for $u$ for $h_{x}=h_{y}=0.05, \operatorname{Re}=10, \Delta t=10^{-4}$ at $t=0.01$ and 1.0. One can see from this table that both the numerical and analytical solutions at selected points at given times are very close to each other. Besides, it is obvious that the computed error norms $L_{2}$ and $L_{\infty}$ are small enough. In a similar way, Table (2) gives the numerical solutions of Example I for $v$ for $h_{x}=h_{y}=0.05, \operatorname{Re}=10, \Delta t=10^{-4}$ at $t=0.01$ and 1.0. One can observe that the numerical results are very close to their exact counterparts and computed error norms are small enough. It is also observed that the numerical solutions found out by the presented scheme are getting closer and closer to analytical values as the mesh sizes are refined.Tables (3-4) present nodal values and the error norms $L_{2}$ and $L_{\infty}$ of $u$ and $v$ but now for 
Table 1. Some nodal values $u$ of Example 1 with the error norms $L_{2}$ and $L_{\infty}$ for $h_{x}=h_{y}=0.05, \operatorname{Re}=10, \Delta t=10^{-4}$ at $t=0.01$ and 1.0 .

\begin{tabular}{clccc}
\hline$(x, y)$ & \multicolumn{2}{c}{$t=0.01$} & \multicolumn{2}{c}{$t=1.0$} \\
\hline & \multicolumn{1}{c}{ Approx. } & Exact & Approx. & Exact \\
\cline { 2 - 5 }$(0.1,0.1)$ & 0.624805 & 0.624805 & 0.605626 & 0.605626 \\
$(0.5,0.1)$ & 0.594202 & 0.594202 & 0.576840 & 0.576840 \\
$(0.9,0.1)$ & 0.567082 & 0.567082 & 0.553017 & 0.553017 \\
$(0.3,0.3)$ & 0.624805 & 0.624805 & 0.605627 & 0.605626 \\
$(0.7,0.3)$ & 0.594202 & 0.594202 & 0.576840 & 0.576840 \\
$(0.1,0.5)$ & 0.655431 & 0.655431 & 0.636685 & 0.636685 \\
$(0.5,0.5)$ & 0.624805 & 0.624805 & 0.605628 & 0.605626 \\
$(0.9,0.5)$ & 0.594202 & 0.594202 & 0.576840 & 0.576840 \\
$(0.3,0.7)$ & 0.655431 & 0.655431 & 0.636687 & 0.636685 \\
$(0.7,0.7)$ & 0.624805 & 0.624805 & 0.605629 & 0.605626 \\
$(0.1,0.9)$ & 0.682611 & 0.682611 & 0.666353 & 0.666353 \\
$(0.5,0.9)$ & 0.655431 & 0.655431 & 0.636687 & 0.636685 \\
$(0.9,0.9)$ & 0.624805 & 0.624805 & 0.605627 & 0.605626 \\
\hline$L_{2}$ & $8.649162 \times 10^{-8}$ & & $2.409775 \times 10^{-6}$ & \\
\hline$L_{\infty}$ & $6.878261 \times 10^{-8}$ & & $2.872069 \times 10^{-6}$ & \\
\hline
\end{tabular}

Table 2. Some nodal values $v$ of Example 1 with the error norms $L_{2}$ and $L_{\infty}$ for $h_{x}=h_{y}=0.05, \operatorname{Re}=10, \Delta t=10^{-4}$ at $t=0.01$ and 1.0 .

\begin{tabular}{ccccc}
\hline$(x, y)$ & \multicolumn{2}{c}{$t=0.01$} & \multicolumn{2}{c}{$t=1.0$} \\
\hline & Approx. & Exact & Approx. & Exact \\
\cline { 2 - 5 }$(0.1,0.1)$ & 0.875195 & 0.875195 & 0.894374 & 0.894374 \\
$(0.5,0.1)$ & 0.905798 & 0.905798 & 0.923160 & 0.923160 \\
$(0.9,0.1)$ & 0.932918 & 0.932918 & 0.946983 & 0.946983 \\
$(0.3,0.3)$ & 0.875195 & 0.875195 & 0.894373 & 0.894374 \\
$(0.7,0.3)$ & 0.905798 & 0.905798 & 0.923160 & 0.923160 \\
$(0.1,0.5)$ & 0.844569 & 0.844569 & 0.863315 & 0.863315 \\
$(0.5,0.5)$ & 0.875195 & 0.875195 & 0.894372 & 0.894374 \\
$(0.9,0.5)$ & 0.905798 & 0.905798 & 0.923160 & 0.923160 \\
$(0.3,0.7)$ & 0.844569 & 0.844569 & 0.863313 & 0.863315 \\
$(0.7,0.7)$ & 0.875195 & 0.875195 & 0.894371 & 0.894374 \\
$(0.1,0.9)$ & 0.817389 & 0.817389 & 0.833647 & 0.833647 \\
$(0.5,0.9)$ & 0.844569 & 0.844569 & 0.863313 & 0.863315 \\
$(0.9,0.9)$ & 0.875195 & 0.875195 & 0.894373 & 0.894374 \\
\hline$L_{2}$ & $6.178088 \times 10^{-8}$ & \multicolumn{3}{c}{$1.637351 \times 10^{-6}$} \\
\hline$L_{\infty}$ & $6.878261 \times 10^{-8}$ & & $2.872070 \times 10^{-6}$ \\
\hline
\end{tabular}

Table 3. Comparison of the approximate and exact solutions $u$ of Example 1 for $h_{x}=h_{y}=0.05, \operatorname{Re}=100$, $\Delta t=10^{-4}$ at $t=0.01$ and 1.0 .

\begin{tabular}{ccccc}
\hline$(x, y)$ & \multicolumn{2}{c}{$t=0.01$} & \multicolumn{2}{c}{$t=1.0$} \\
\hline & Approx. & Exact & Approx. & Exact \\
\cline { 2 - 5 }$(0.1,0.1)$ & 0.623106 & 0.623047 & 0.510307 & 0.510522 \\
$(0.5,0.1)$ & 0.501617 & 0.501622 & 0.500072 & 0.500074 \\
$(0.9,0.1)$ & 0.500011 & 0.500011 & 0.500000 & 0.500000 \\
$(0.3,0.3)$ & 0.623106 & 0.623047 & 0.509823 & 0.510522 \\
$(0.7,0.3)$ & 0.501617 & 0.501622 & 0.500067 & 0.500074 \\
$(0.1,0.5)$ & 0.748272 & 0.748274 & 0.716948 & 0.716759 \\
$(0.5,0.5)$ & 0.623106 & 0.623047 & 0.509497 & 0.510522 \\
$(0.9,0.5)$ & 0.501617 & 0.501622 & 0.500063 & 0.500074 \\
$(0.3,0.7)$ & 0.748272 & 0.748274 & 0.717267 & 0.716759 \\
$(0.7,0.7)$ & 0.623106 & 0.623047 & 0.509311 & 0.510522 \\
$(0.1,0.9)$ & 0.749988 & 0.749988 & 0.749738 & 0.749742 \\
$(0.5,0.9)$ & 0.748272 & 0.748274 & 0.717532 & 0.716759 \\
$(0.9,0.9)$ & 0.623106 & 0.623047 & 0.509170 & 0.510522 \\
\hline$L_{2}$ & $3.822706 \times 10^{-5}$ & \multicolumn{3}{c}{$1.341393 \times 10^{-3}$} \\
\hline$L_{\infty}$ & $6.086191 \times 10^{-5}$ & & $2.903955 \times 10^{-3}$ \\
\hline
\end{tabular}


Table 4. Comparison of the approximate and exact solutions $v$ of Example 1 for $h_{x}=h_{y}=0.05, \operatorname{Re}=100$, $\Delta t=10^{-4}$ at $t=0.01$ and 1.0 .

\begin{tabular}{ccccc}
\hline$(x, y)$ & \multicolumn{2}{c}{$t=0.01$} & \multicolumn{2}{c}{$t=1.0$} \\
\hline & Approx. & Exact & Approx. & Exact \\
\cline { 2 - 5 }$(0.1,0.1)$ & 0.876894 & 0.876953 & 0.989693 & 0.989478 \\
$(0.5,0.1)$ & 0.998383 & 0.998378 & 0.999928 & 0.999926 \\
$(0.9,0.1)$ & 0.999989 & 0.999989 & 1.000000 & 1.000000 \\
$(0.3,0.3)$ & 0.876894 & 0.876953 & 0.990177 & 0.989478 \\
$(0.7,0.3)$ & 0.998383 & 0.998378 & 0.999933 & 0.999926 \\
$(0.1,0.5)$ & 0.751728 & 0.751726 & 0.783052 & 0.783241 \\
$(0.5,0.5)$ & 0.876894 & 0.876953 & 0.990503 & 0.989478 \\
$(0.9,0.5)$ & 0.998383 & 0.998378 & 0.999937 & 0.999926 \\
$(0.3,0.7)$ & 0.751728 & 0.751726 & 0.782733 & 0.783241 \\
$(0.7,0.7)$ & 0.876894 & 0.876953 & 0.990689 & 0.989478 \\
$(0.1,0.9)$ & 0.750012 & 0.750012 & 0.750262 & 0.750258 \\
$(0.5,0.9)$ & 0.751728 & 0.751726 & 0.782468 & 0.783241 \\
$(0.9,0.9)$ & 0.876894 & 0.876953 & 0.990830 & 0.989478 \\
\hline$L_{2}$ & $2.744679 \times 10^{-5}$ & \multicolumn{3}{c}{$8.302825 \times 10^{-4}$} \\
\hline$L_{\infty}$ & $6.086191 \times 10^{-5}$ & \multicolumn{3}{c}{$2.903955 \times 10^{-3}$} \\
\hline
\end{tabular}

a larger value of Reynold number $\mathrm{Re}=100$, respectively. From those tables one can see that both of the error norms increase as the Reynold number increases. In the Figures (1-2), one can see first exact and then numerical solutions for $u$ and $v$ of Example 1 for values of $h_{x}=h_{y}=0.05, \operatorname{Re}=100, \Delta t=10^{-4}$ at $t=0.5$,respectively.

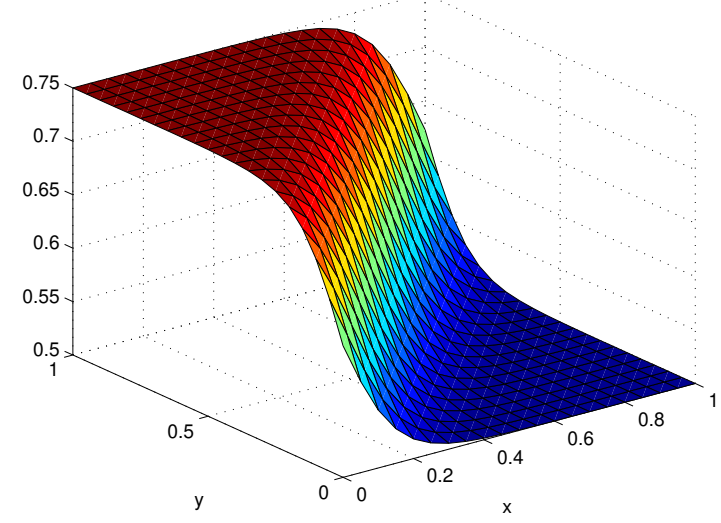

(a)

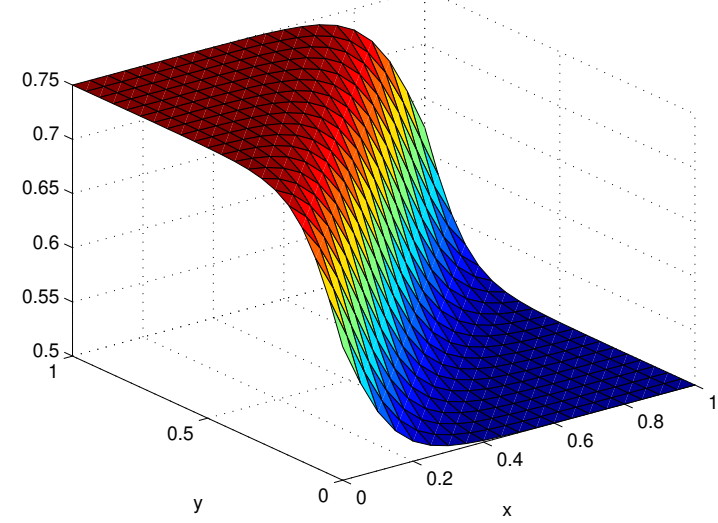

(b)

Figure 1. The profiles of Example 1 of (a) exact and (b) numerical solutions for $u$ for $h_{x}=h_{y}=0.05, \operatorname{Re}=100$, $\Delta t=10^{-4}$ at $t=0.5$. 
Table 5. A comparison of numerical solutions for $u$ of Example 2 with those given in $[6,7,13]$ for $h_{x}=h_{y}=0.025$, $\operatorname{Re}=500, \Delta t=10^{-4}$ at $t=0.625$.

\begin{tabular}{cccccc}
\hline$(x, y)$ & \multicolumn{5}{c}{$u$} \\
\hline & Present & {$[6]$} & {$[6] \mathrm{N}=40$} & {$[7]$} & {$[13]$} \\
\cline { 2 - 6 }$(0.15,0.1)$ & 0.96870 & 0.95691 & 0.96066 & 0.96650 & 0.96870 \\
$(0.3,0.1)$ & 1.03202 & 0.95616 & 0.96852 & 1.02970 & 1.03200 \\
$(0.1,0.2)$ & 0.84619 & 0.84257 & 0.84104 & 0.84449 & 0.86178 \\
$(0.2,0.2)$ & 0.87814 & 0.86399 & 0.86866 & 0.87631 & 0.87813 \\
$(0.1,0.3)$ & 0.67920 & 0.67667 & 0.67792 & 0.67809 & 0.67920 \\
$(0.3,0.3)$ & 0.79947 & 0.76876 & 0.77254 & 0.79792 & 0.79945 \\
$(0.15,0.4)$ & 0.54674 & 0.54408 & 0.54543 & 0.54601 & 0.66039 \\
$(0.2,0.4)$ & 0.58959 & 0.58778 & 0.58564 & 0.58874 & 0.58958 \\
\hline
\end{tabular}

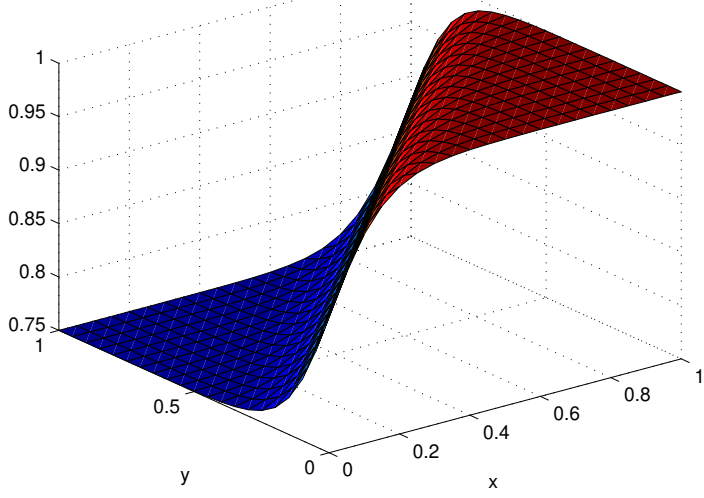

(a)

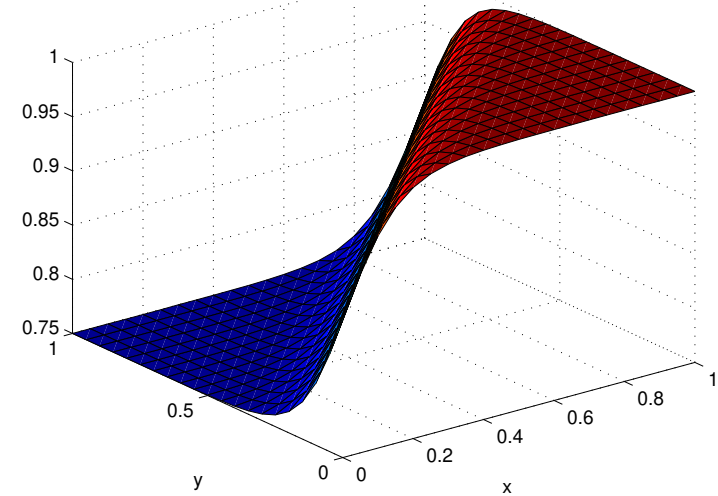

(b)

Figure 2. The profiles of Example 1 of (a) exact and (b) numerical solutions for $v$ for values of $h_{x}=h_{y}=0.05$, $\operatorname{Re}=100, \Delta t=10^{-4}$ at $t=0.5$.

Example II: Secondly, Crank-Nicolson finite difference method has been applied to 2D-cBE on the solution domain $\Omega=[0,0.5] \times[0,0.5]$ with the following initial

$$
u(x, y, 0)=\sin \pi x+\cos \pi y, v(x, y, 0)=x+y
$$

and boundary conditions

$$
\begin{aligned}
& \left.\begin{array}{cc}
u(0, y, t)=\cos (\pi y), & u(0.5, y, t)=1+\cos (\pi y) \\
v(0, y, t)=y, & v(0.5, y, t)=0.5+y
\end{array}\right\} 0 \leq y \leq 0.5, t \geq 0, \\
& \left.\begin{array}{cc}
u(x, 0, t)=1+\sin (\pi x) & u(x, 0.5, t)=\sin (\pi x) \\
v(x, 0, t)=x & v(x, 0.5, t)=x+0.5
\end{array}\right\} 0 \leq x \leq 0.5, t \geq 0 .
\end{aligned}
$$

[13]. Unlike the Example I, Example II has no analytical solution. Due to this fact, Table (5) only gives a comparison of numerical solutions for $u$ of Example II for $h_{x}=h_{y}=0.025$, $\operatorname{Re}=500, \Delta t=10^{-4}$ at $t=0.625$ with those in Refs. $[6,7,13]$. Again, due to the same reason, Table (6) presents a comparison of numerical solutions for $v$ of Example II for values of $h_{x}=h_{y}=0.025, \operatorname{Re}=500, \Delta t=10^{-4}$ at time $t=0.625$ with those in Refs. [6, 7, 13]. Tables (7-8) show also pointwise values of $u$ and $v$ but now for a smaller value of $\operatorname{Re}=50$, respectively. Figures (3) shows numerical solutions of $u$ and $v$ of Example II for $h_{x}=h_{y}=0.025, \operatorname{Re}=50, \Delta t=10^{-4}$ at $t=0.625$, respectively. 
Table 6. A comparison of numerical solutions for $v$ of Example 2 with those given in $[6,7,13]$ for $h_{x}=h_{y}=0.025$, $\operatorname{Re}=500, \Delta t=10^{-4}$ at $t=0.625$.

\begin{tabular}{cccccc}
\hline$(x, y)$ & \multicolumn{5}{c}{$v$} \\
\hline & Present & {$[6]$} & {$[6] \mathrm{N}=40$} & {$[7]$} & {$[13]$} \\
\cline { 2 - 6 }$(0.15,0.1)$ & 0.09043 & 0.10177 & 0.08612 & 0.09020 & 0.09043 \\
$(0.3,0.1)$ & 0.10728 & 0.13287 & 0.07712 & 0.10690 & 0.10728 \\
$(0.1,0.2)$ & 0.18010 & 0.18503 & 0.17828 & 0.17972 & 0.17295 \\
$(0.2,0.2)$ & 0.16816 & 0.18169 & 0.16202 & 0.16777 & 0.16816 \\
$(0.1,0.3)$ & 0.26268 & 0.26560 & 0.26094 & 0.26222 & 0.26268 \\
$(0.3,0.3)$ & 0.23550 & 0.25142 & 0.21542 & 0.23497 & 0.23550 \\
$(0.15,0.4)$ & 0.31799 & 0.32084 & 0.31360 & 0.31753 & 0.29022 \\
$(0.2,0.4)$ & 0.30419 & 0.30927 & 0.29776 & 0.30371 & 0.30418 \\
\hline
\end{tabular}

Table 7. A comparison of numerical solutions for $u$ of Example 2 with those given in $[6,7,13]$ for $h_{x}=h_{y}=0.025$, $\operatorname{Re}=50, \Delta t=10^{-4}$ at $t=0.625$.

\begin{tabular}{cllll}
\hline$(x, y)$ & \multicolumn{4}{c}{$u$} \\
\hline & Present & {$[6]$} & {$[7]$} & {$[13]$} \\
\cline { 2 - 5 }$(0.1,0.1)$ & 0.97146 & 0.97258 & 0.96688 & 0.97146 \\
$(0.3,0.1)$ & 1.15282 & 1.16214 & 1.14827 & 1.15280 \\
$(0.2,0.2)$ & 0.86307 & 0.86281 & 0.85911 & 0.86308 \\
$(0.4,0.2)$ & 0.97981 & 0.96483 & 0.97637 & 0.97984 \\
$(0.1,0.3)$ & 0.66316 & 0.66318 & 0.66019 & 0.66316 \\
$(0.3,0.3)$ & 0.77230 & 0.77030 & 0.76932 & 0.77232 \\
$(0.2,0.4)$ & 0.58180 & 0.58070 & 0.57966 & 0.58181 \\
$(0.4,0.4)$ & 0.75855 & 0.74435 & 0.75678 & 0.75860 \\
\hline
\end{tabular}

Table 8. A comparison of numerical solutions for $v$ of Example 2 with those given in $[6,7,13]$ for $h_{x}=h_{y}=0.025$, $\operatorname{Re}=50, \Delta t=10^{-4}$ at $t=0.625$.

\begin{tabular}{cllll}
\hline$(x, y)$ & \multicolumn{4}{l}{$v$} \\
\hline & Present & {$[6]$} & {$[7]$} & {$[13]$} \\
\cline { 2 - 5 }$(0.1,0.1)$ & 0.09869 & 0.09773 & 0.09824 & 0.09869 \\
$(0.3,0.1)$ & 0.14158 & 0.14039 & 0.14112 & 0.14158 \\
$(0.2,0.2)$ & 0.16754 & 0.16660 & 0.16681 & 0.16754 \\
$(0.4,0.2)$ & 0.17109 & 0.17397 & 0.17065 & 0.17110 \\
$(0.1,0.3)$ & 0.26378 & 0.26294 & 0.26261 & 0.26378 \\
$(0.3,0.3)$ & 0.22654 & 0.22463 & 0.22576 & 0.22655 \\
$(0.2,0.4)$ & 0.32851 & 0.32402 & 0.32745 & 0.32851 \\
$(0.4,0.4)$ & 0.32499 & 0.31822 & 0.32441 & 0.32501 \\
\hline
\end{tabular}


Table 9. Some nodal values $u$ of Example 3 with the error norms $L_{2}$ and $L_{\infty}$ for $h_{x}=h_{y}=0.05, \operatorname{Re}=1000$, $\Delta t=10^{-3}$ at $t=0.01,0.5$ and 1.0 .

\begin{tabular}{crrrrrr}
\hline$(x, y)$ & \multicolumn{2}{c}{$t=0.01$} & \multicolumn{2}{c}{$t=0.5$} & \multicolumn{2}{c}{$t=1.0$} \\
\hline & \multicolumn{1}{c}{ Approx. } & \multicolumn{1}{l}{ Exact } & Approx. & \multicolumn{1}{l}{ Exact } & \multicolumn{1}{l}{ Approx. } & \multicolumn{1}{l}{ Exact } \\
\cline { 2 - 7 }$(0.1,0.1)$ & -0.001439 & -0.001439 & -0.001408 & -0.001408 & -0.001376 & -0.001376 \\
$(0.5,0.1)$ & 0.001941 & 0.001941 & 0.001895 & 0.001894 & 0.001849 & 0.001848 \\
$(0.9,0.1)$ & -0.001727 & -0.001727 & -0.001682 & -0.001682 & -0.001638 & -0.001637 \\
$(0.3,0.3)$ & 0.001134 & 0.001134 & 0.001114 & 0.001114 & 0.001094 & 0.001094 \\
$(0.7,0.3)$ & 0.002551 & 0.002551 & 0.002458 & 0.002453 & 0.002368 & 0.002359 \\
$(0.1,0.5)$ & -0.003927 & -0.003927 & -0.003854 & -0.003854 & -0.003780 & -0.003781 \\
$(0.5,0.5)$ & 0.006280 & 0.006280 & 0.006130 & 0.006130 & 0.005981 & 0.005981 \\
$(0.9,0.5)$ & -0.007194 & -0.007194 & -0.006960 & -0.006953 & -0.006731 & -0.006718 \\
$(0.3,0.7)$ & 0.001134 & 0.001134 & 0.001114 & 0.001114 & 0.001094 & 0.001094 \\
$(0.7,0.7)$ & 0.002551 & 0.002551 & 0.002458 & 0.002453 & 0.002368 & 0.002359 \\
$(0.1,0.9)$ & -0.001439 & -0.001439 & -0.001408 & -0.001408 & -0.001376 & -0.001376 \\
$(0.5,0.9)$ & 0.001941 & 0.001941 & 0.001895 & 0.001894 & 0.001849 & 0.001848 \\
$(0.9,0.9)$ & -0.001727 & -0.001727 & -0.001682 & -0.001682 & -0.001638 & -0.001637 \\
\hline$L_{2}$ & $2.2082 \times 10^{-5}$ & \multicolumn{7}{c}{$1.0301 \times 10^{-3}$} & & $1.9265 \times 10^{-3}$ & \\
\hline$L_{\infty}$ & $2.8221 \times 10^{-7}$ & \multicolumn{7}{c}{$1.2650 \times 10^{-5}$} & & $2.2915 \times 10^{-5}$ & \\
\hline
\end{tabular}

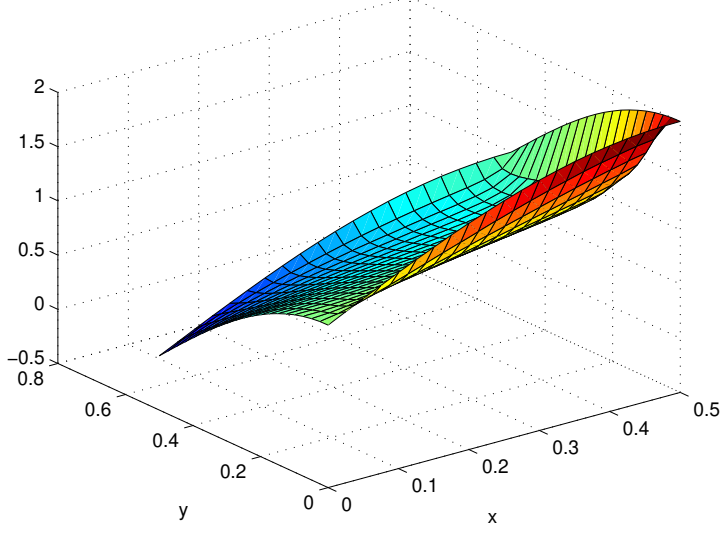

(a)

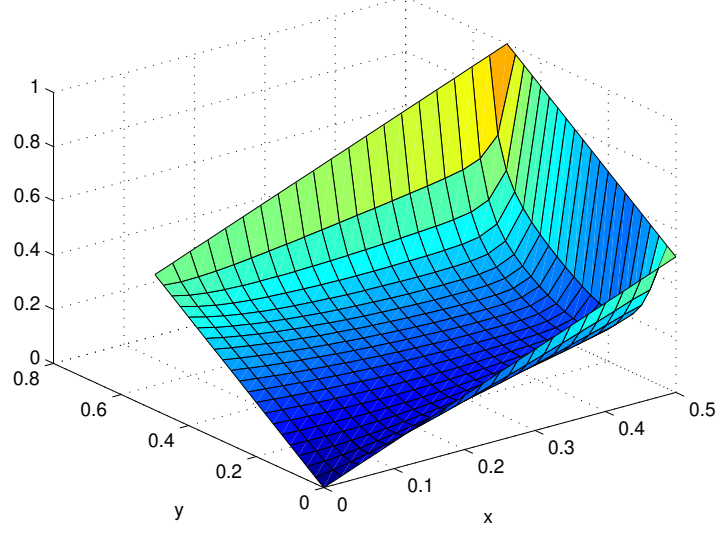

(b)

Figure 3. The numerical profiles of (a) $u$ and (b) $v$ of Example 2 for $h_{x}=h_{y}=0.025, \operatorname{Re}=50, \Delta t=10^{-4}$ at $t=0.625$.

Example III: Thirdly, the solution domain is taken as $\Omega=\{(x, y): 0 \leq x \leq 1,0 \leq y \leq 1\}$ and its exact solution is [13]

$$
\begin{aligned}
& u(x, y, t)=-\frac{4 \pi e^{-\frac{5 \pi^{2} t}{\mathrm{Re}}} \cos (2 \pi x) \sin (\pi y)}{\operatorname{Re}\left(2+e^{-\frac{5 \pi^{2} t}{\mathrm{Re}}} \sin (2 \pi x) \sin (\pi y)\right.}, \\
& v(x, y, t)=-\frac{2 \pi e^{-\frac{5 \pi^{2} t}{\mathrm{Re}}} \sin (2 \pi x) \cos (\pi y)}{\operatorname{Re}\left(2+e^{-\frac{5 \pi^{2} t}{\mathrm{Re}}} \sin (2 \pi x) \sin (\pi y)\right.},
\end{aligned}
$$

Table (9) gives approximate solutions of $u$ of Example III for $h_{x}=h_{y}=0.05, \operatorname{Re}=1000, \Delta t=10^{-3}$ at $t=0.01,0.5$ ve 1.0. From the table one can easily see that the approximate and exact solutions are very close to each other and calculated error norms $L_{2}$ and $L_{\infty}$ are small enough. In a similar manner, Table (10) presents numerical solutions of $v$ of Example 3 for values of $h_{x}=h_{y}=0.05, \mathrm{Re}=1000, \Delta t=10^{-3}$ at times $t=0.01,0.5$ ve 1.0. Again, one can see from this table that both of the approximate and exact pointwise values are in good agreement. Th error norms $L_{2}$ and $L_{\infty}$ show the general consistency between the approximate and exact solutions throughout the solution domain. Figures (4-5) show (a) exact and (b) approximate solutions for $u$ and $v$ of Example 3 for values of $h_{x}=h_{y}=0.05$, $\operatorname{Re}=1000, \Delta t=10^{-3}$ at $t=0.01$,respectively. 
Table 10. Some nodal values $v$ of Example 3 with the error norms $L_{2}$ and $L_{\infty}$ for $h_{x}=h_{y}=0.05, \operatorname{Re}=1000$, $\Delta t=10^{-3}$ at $t=0.01,0.5$ and 1.0 .

\begin{tabular}{|c|c|c|c|c|c|c|}
\hline$(x, y)$ & \multicolumn{2}{|c|}{$t=0.01$} & \multicolumn{2}{|c|}{$t=0.5$} & \multicolumn{2}{|c|}{$t=1.0$} \\
\hline & Approx. & Exact & Approx. & Exact & Approx. & Ecaxt \\
\hline$(0.1,0.1)$ & -0.001609 & -0.001609 & -0.001574 & -0.001574 & -0.001539 & -0.001539 \\
\hline$(0.5,0.1)$ & -0.000000 & -0.000000 & -0.000000 & -0.000000 & -0.000001 & -0.000000 \\
\hline$(0.9,0.1)$ & 0.001931 & 0.001931 & 0.001880 & 0.001880 & 0.001830 & 0.001830 \\
\hline$(0.3,0.3)$ & -0.001268 & -0.001268 & -0.001246 & -0.001246 & -0.001223 & -0.001224 \\
\hline$(0.7,0.3)$ & 0.002852 & 0.002852 & 0.002743 & 0.002743 & 0.002643 & 0.002637 \\
\hline$(0.1,0.5)$ & -0.000000 & -0.000000 & -0.000000 & -0.000000 & 0.000000 & -0.000000 \\
\hline$(0.5,0.5)$ & -0.000000 & -0.000000 & 0.000000 & -0.000000 & -0.000000 & -0.000000 \\
\hline$(0.9,0.5)$ & 0.000000 & 0.000000 & 0.000000 & 0.000000 & 0.000000 & 0.000000 \\
\hline$(0.3,0.7)$ & 0.001268 & 0.001268 & 0.001246 & 0.001246 & 0.001223 & 0.001224 \\
\hline$(0.7,0.7)$ & -0.002852 & -0.002852 & -0.002746 & -0.002743 & -0.002643 & -0.002637 \\
\hline$(0.1,0.9)$ & 0.001609 & 0.001609 & 0.001574 & 0.001574 & 0.001539 & 0.001539 \\
\hline$(0.5,0.9)$ & 0.000000 & 0.000000 & 0.000000 & 0.000000 & 0.000001 & 0.000000 \\
\hline$(0.9,0.9)$ & -0.001931 & -0.001931 & -0.001880 & -0.001880 & -0.001830 & -0.001830 \\
\hline$L_{2}$ & $1.2840 \times 10^{-5}$ & & $6.0180 \times 10^{-4}$ & & $1.1312 \times 10^{-3}$ & \\
\hline$L_{\infty}$ & $9.3384 \times 10^{-8}$ & & $4.1425 \times 10^{-6}$ & & $7.3706 \times 10^{-6}$ & \\
\hline
\end{tabular}

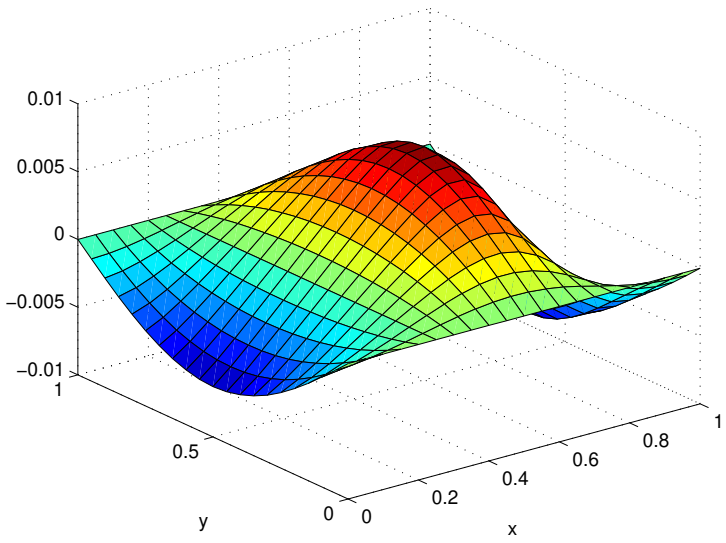

(a)

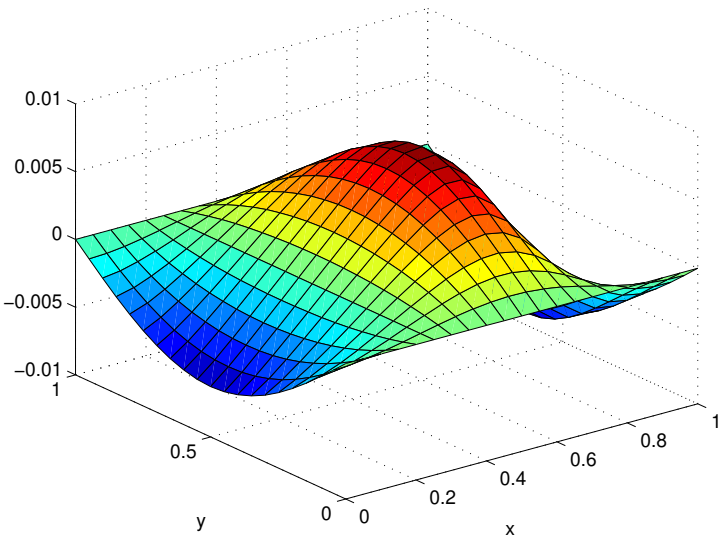

(b)

Figure 4. The profiles of Example 3 of (a) exact and (b) numerical solutions of $u$ for $h_{x}=h_{y}=0.05, \operatorname{Re}=1000$, $\Delta t=10^{-3}$ at $t=0.01$.

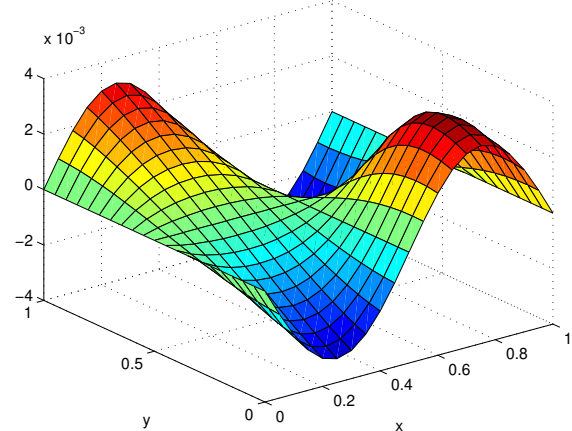

(a)

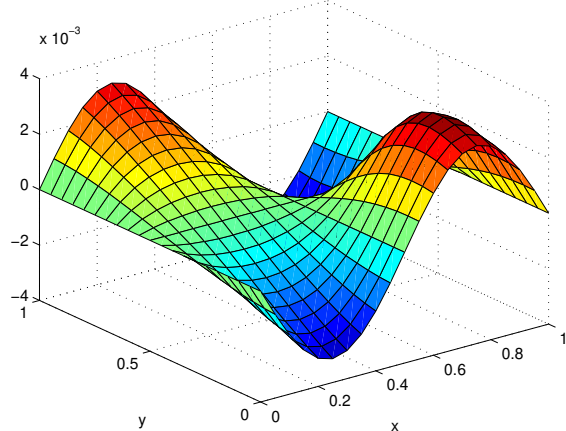

(b)

Figure 5. The profiles of Example 3 of (a) exact and (b) numerical solutions of $v$ for $h_{x}=h_{y}=0.05, \operatorname{Re}=1000$, $\Delta t=10^{-3}$ at $t=0.01$. 


\section{Conclusion}

In this study, the proposed scheme resulting an implicit linear algebraic system has been successfully applied to obtain the approximate solutions of two dimensional Burgers equation. The error norms $L_{2}$ and $L_{\infty}$ of the presented scheme are calculated. The three numerical experiments showed that the approximate solutions are in very good agreement with the analytical ones, and also the error norms are adequately small. The obtained results support that the numerical accuracy of the scheme is in consistency with its theoretical value and that the scheme is also unconditionally stable. In conclusion, the present numerical scheme, which can be easily implemented, produces accurate and reliable results. As a future work, the method can be successfully used to find approximate solutions of such combined partial differential equations that play an important role in describing nonlinear wave propagation encountered in physics and applied mathematics.

\section{Acknowledgment.}

We thank the reviewer for their insightful comments and suggestions that helped us improve the paper.

\section{Funding}

There is no funding for this work.

\section{Availability of data and materials}

Not applicable.

\section{Competing interests}

The authors declare that they have no competing interests.

\section{Author's contributions}

All authors contributed equally to the writing of this paper. All authors read and approved the final manuscript.

\section{References}

[1] Fletcher, C. A. J.: Generating exact solutions of the two-dimensional Burgers' equations. International Journal for Numerical Methods in Fluids. 3, 213-216 (1983). https:/ / doi.org/10.1002/fld.1650030302

[2] Yagmurlu, N. M., Gagir A.: Numerical Simulation of Two Dimensional Coupled Burgers Equations by Rubin-Graves Type Linearization. Mathematical Sciences and Applications E-notes. 9 (4), 158-169 (2021). https://doi.org/10.36753/mathenot.947552

[3] Fletcher, C. A. J.: A comparison of finite element and finite difference solutions of the one- and two-dimensional Burgers' equations. Journal of Computational Physics. 51 (1), 159-188 (1983). https://doi.org/10.1016/00219991(83)90085-2

[4] Goyon, O.: Multilevel Schemes for Solving Unsteady Equations. International Journal for Numerical Methods in Fluids. 22 (10), 937-959 (1996).

[5] Arshed A., Siraj-ul-Islam, Sirajul H.: A Computational Meshfree Technique for the Numerical Solution of the TwoDimensional Coupled Burgers' Equations. International Journal for Computational Methods in Engineering Science and Mechanics. 10 (5), 406-422 (2009). https:/ / doi.org/10.1080/15502280903108016

[6] Jain, P. C., Holla, D. N.: Numerical solutions of coupled Burgers' equation. International Journal of Non-Linear. Meechanics. 13 (4), 213-222 (1978), https:/ / doi.org/10.1016/0020-7462(78)90024-0.

[7] Bahadır, A. R.: A fully implicit finite-difference scheme for two-dimensional Burgers' equations. Applied Mathematics and Computation. 137 (1), 131-137 (2003). https:/ / doi.org/10.1016/S0096-3003(02)00091-7 
[8] Khater, A. H., Temsah, R. S, Hassan, M.M.: Chebyshev spectral collocation method for solving Burgers'-type equations. Journal of Computational and Applied Mathematics. 222 (2) 333-350 (2008). https://doi.org/10.1016/j.cam.2007.11.007https://doi.org/10.1016/j.cam.200711.007

[9] Mittal, R. C., Jiwari, R.: Differential Quadrature Method for Two-Dimensional Burgers' Equations. International Journal for Computational Methods in Engineering Science and Mechanics. 10 (6), 450-459 (2009). https:/ /doi:10.1080/15502280903111424

[10] Liao, W.: A fourth-order finite-difference method for solving the system of two-dimensional Burgers' equations. International Jlournal Numerical Methods in Fluids. 64 (5) 565-590 (2010). https:/ / doi.org/10.1002/fld.2163

[11] Zhu, H., Shu, H., Ding, M.: Numerical solutions of two-dimensional Burgers' equations by discrete Adomian decomposition method. Computers and Mathematics with Applications. 60 (3) 840-848 (2010). https://doi.org/10.1016/j.camwa.2010.05.031

[12] Srivastava, V. K., Tamsir, M., Bhardwaj, U., Sanyasiraju, Y.: Crank-Nicolson Scheme for Numerical Solutions of Two-dimensional Coupled Burgers' Equations. International Journal of Scientific \& Engineering Research. 2 (5), 1-6 (2011).

[13] Tamsir, M., Srivastava, V. K.: A semi-implicit finite-difference approach for two-dimensional coupled Burgers equations. International Journal of Scientific \& Engineering Research. 2 (6), 46-51 (2011). ISSN 2229-5518

[14] Srivastava, V. K., Tamsir, M.: Crank-Nicolson Semi-Implicit Approach For Numerical Solutions of Two- Dimensional Coupled Nonlinear Burgers Equations. International Journal of Applied Mechanics and Engineering. 17 (2), 571-581 (2012).

[15] Thakar, S., Wani, S.: Linear Method For Two Dimensional Burgers Equation. Ultra Scientist. 25 (1)A, 156-168 (2013).

[16] Srivastava, V. K., Awasthi, M.K., Singh, S.: An implicit logarithmic finite-difference technique for two dimensional coupled viscous Burgers' equation. AIP Advances. 3 (12), 122105 (2013). https:/ /doi:10.1063/1.4842595

[17] Srivastava, V. K., Singh, S., Awasthi, M. K.: Numerical solutions of coupled Burgers equations by an implicit finite difference scheme. AIP Advances. 3 (8), 082131 (2013). https:/ / doi: 10.1063/1.4820355

[18] Srivastava, V. K., Singh, B. K.: A robust finite difference scheme for the numerical solutions of two dimensional time dependent coupled nonlinear Burgers equations. International Journal of Applied Mathematics and Mechanics. 10 (7), 28-39 (2014).

[19] Zhang, L., Wang, L., Ding, X.:Exact finite-difference scheme and nonstandard finite-difference scheme for coupled Burgers equation. Advances in Difference Equations. 122 (2014) (2014). https://doi:10.1186/1687-1847-2014-122

[20] Mittal, R. C., Tripathi, A.: Numerical solutions of two-dimensional Burgers' equations using modified Bi-cubic B-spline finite elements. Engineering Computations. 32 (5), 1275 - 1306.(2015). https:/ / doi.org/10.1108/EC-04-2014-0067

[21] Tamsir, M., Srivastava, V. K., Jiwari, R.: An algorithm based on exponential modified cubic B-spline differential quadrature method for nonlinear Burgers' equation. Applied Mathematics and Computation. 290, 111-124 (2016). https://doi.org/10.1016/j.amc.2016.05.048

[22] Zhanlav, T., Chuluunbaatar, O., Ulziibayar, V.: Higher-Order Numerical Solution of Two-Dimensional Coupled Burgers Equations. American Journal of Computational Mathematics. 6 (2), 120-129 (2016). https://doi:10.4236/ajcm.2016.62013

[23] Ngondiep, E.: An efficient three-level explicit time-split scheme for solving two-dimensional unsteady nonlinear coupled Burgers' equations. Int J Numer Meth Fluids. 92 (4), 266-284 (2020). https:/ / doi.org/10.1002/fld.4783

[24] Saqib, M., Hasnain, S., Mashat, D. S.: Highly Efficient Computational Methods for Two Dimensional Coupled Nonlinear Unsteady Convection-Diffusion Problems. IEEE Access. 5, 7139-7148 (2017). https://doi:10.1109/ACCESS.2017.2699320

[25] Wubs, F. W., de Goede, E. D.: An explicit-implicit method for a class of time-dependent partial differential equations. Appl. Numer. Math. 9 (2), 157-181 (1992). https:/ / doi.org/10.1016/0168-9274(92)90012-3 
[26] Kutluay, S., Yağmurlu, N. M.: The Modified Bi-quintic B-Splines for Solving the Two-Dimensional Unsteady Burgers' Equation. European International Journal of Science and Technology. 1(2), 23-39 (2012).

[27] Başhan, A.: A numerical treatment of the coupled viscous Burgers' equation in the presence of very large Reynolds number. Physica A: Statistical Mechanics and its Applications. 545, (2020). https://doi.org/10.1016/j.physa.2019.123755

[28] Başhan, A., Karakoç, S. B. G., Geyikli, T.: B-spline Differential Quadrature Method for the Modified Burgers' Equation. Çankaya University Journal of Science and Engineering. 12 (1), 001-013 (2015) .

[29] Uçar, Y., Yağmurlu, N. M., Başhan, A.: Numerical Solutions and Stability Analysis of Modified Burgers Equation via Modified Cubic B-Spline Differential Quadrature Methods. Sigma J Eng \& Nat Sci. 37 (1), 129-142 (2019).

[30] Karakoç, S. B. G., Başhan, A., Geyikli, T.: Two Different Methods for Numerical Solution of the Modified Burgers' Equation. The Scientific World Journal. 2014 (5), 1-13 (2014). https:/ / doi.org/10.1155/2014/780269.

[31] Başhan, A.: Nonlinear dynamics of the Burgers' equation and numerical experiments. Math Sci (2021). https://doi.org/10.1007/s40096-021-00410-8.

[32] Karakoç, B., Bhowmik, S. K.: Galerkin Finite Element Solution for Benjamin-Bona-Mahony-Burgers Equation with Cubic B-Splines. Computers and Mathematics with Applications. 77 (7), 1917-1932 (2019).

[33] Bhowmik, S. K., Karakoç, S. B. G.: Numerical approximation of the generalized regularized long wave equation using Petrov Galerkin finite element method. Numerical Methods for Partial Differential Equations. 35 (6), 2236-2257 (2019).

[34] Chai, Y., Ouyang, J.: Appropriate stabilized Galerkin approaches for solving two-dimensional coupled Burgers' equations at high Reynolds numbers. Computers and Mathematics with Applications. 79 (5), 1287-1301 (2020). https://doi.org/10.1016/j.camwa.2019.08.036

[35] Shukla, H. S., Tamsir, M., Srivastava, V. K., Kumar, J.: Numerical Solution of two dimensional coupled viscous Burgers' Equation using the Modified Cubic B-Spline Differential Quadrature Method. AIP Advances. 4 (11), 1-10 (2014).https://doi.org/10.1063/1.4902507

\section{Affiliations}

NURI MURAT YAĞMURLU

ADDRESS: Inonu University, Dept. of Mathematics, 44200, Malatya-TURKEY.

E-MAIL: murat.yagmurlu@inonu.edu.tr

ORCID ID: 0000-0003-1593-0254

ABDULNASIR GAGIR

AdDrEss: Inonu University, Dept. of Mathematics, 44200, Malatya-TURKEY.

E-MAIL: ansrggr@gmail.com

ORCID ID: 0000-0003-1029-4447 